10-2007

\title{
On the Reference Length and Mode Mixity for a Bimaterial Interface
}

A. Agrawal

University of Delaware

Anette M. Karlsson

Cleveland State University, a.karlsson@csuohio.edu

Follow this and additional works at: https://engagedscholarship.csuohio.edu/enme_facpub

Part of the Mechanical Engineering Commons

How does access to this work benefit you? Let us know!

\section{Original Citation}

Agrawal, A., and Karlsson, A. M., 2007, "On the Reference Length and Mode Mixity for a Bimaterial Interface," Journal of Engineering Materials and Technology, 129(4) pp. 580-587.

This Article is brought to you for free and open access by the Mechanical Engineering Department at EngagedScholarship@CSU. It has been accepted for inclusion in Mechanical Engineering Faculty Publications by an authorized administrator of EngagedScholarship@CSU. For more information, please contact library.es@csuohio.edu. 


\section{On the Reference Length and Mode Mixity for a Bimaterial Interface}

\author{
A. M. Karlsson ${ }^{1}$ \\ e-mail: karlsson@udel.edu \\ Department of Mechanical Engineering, \\ University of Delaware, \\ Newark, DE 19716
}

We investigate properties that govern interfacial fracture within the framework of linear elastic fracture mechanics, including interfacial fracture toughness, mode mixity, and the associated reference length. The reference length describes the arbitrary location where the mode mixity is evaluated, ahead of the crack tip, in a bimaterial system. A method for establishing a reference length that is fixed for a given bimaterial system is proposed. This is referred to as the "characteristic reference length," with the associated "characteristic mode mixity." The proposed method is illustrated with an experimental investigation, utilizing a four-point bend test of a bimaterial system.

Keywords: interface crack, oscillatory singularity, mode mixity, reference length, interfacial toughness

\section{Introduction}

Interfaces between two dissimilar materials are common in engineering design, including diverse applications such as fiberreinforced composites, microelectronics, coatings, and adhesive joints. To ensure reliable structures, it is pertinent to understand the failure mechanics of the bimaterial interfaces that are associated with such structures, including interfacial cracking, e.g., $[1,2]$. Compared to fracture of homogeneous materials-where cracks tend to propagate in pure mode I (tensile load) - interfacial cracks propagate along the interface (direction of weakest path) under mixed mode conditions (combination of modes I and II, i.e, tensile and shear load).

The fracture resistance of the interface can be quantified by an energy functional, the interfacial fracture toughness $\Gamma_{\text {int }}$. The contributions to $\Gamma_{\text {int }}$ come from the work of adhesion and the dissipative work. The dissipative work (e.g., plasticity, roughness, and shielding effects of the interface) increases with mode II loading and can be significantly larger than the work of adhesion $[1,3]$. Accordingly, experimental observations suggest that the interfacial fracture toughness is significantly enhanced in the presence of shear load [1-5]. Thus, $\Gamma_{\text {int }}$ is governed by the mode mixity, which relates the relative proportions of the sliding and the opening modes ahead of the crack tip $[1,2]$. The interfacial fracture is commonly characterized by a toughness curve, expressing the interfacial fracture toughness as a function of mode mixity, e.g., [6-19]. The toughness curve is a property of the bimaterial interface and should be invariant of the specimen type or specimen geometry $[1,2,7-9]$. For bimaterial interfaces, the mode mixity is specified at a particular distance ahead of the crack tip, referred to as the reference length. Thus, mode mixity and, hence, the reference length are, along with the interfacial fracture toughness, important parameters for interfacial fracture.

Even though interfacial fracture mechanics has received significant attention, e.g., [20-30], there are still unresolved issues relating to determining mode mixity and reference length. In this paper, we will address the issue of how the reference length can appropriately be selected. To this end, the paper is outlined as follows: In Sec. 2, we will review the fundamental fracture mechanics parameters, including the need and definition of the ref-

${ }^{1}$ Corresponding author. erence length. In Sec. 3, we will propose a method on how to establish an appropriate reference length for a material system. We will refer to this material-based reference length as the characteristic reference length $\hat{l}_{c}$, and the associated mode mixity as the characteristic mode mixity $\psi_{c}$. With that established, we will explore the proposed methodology for a set of test specimens. As will be evident later, we consider a "local mode mixity," representing the state in the vicinity of the crack tip, and not the "global mode mixity," which depends to the far field (applied) stress. Thus, within the formulation of the problem, we do not distinguish between long and short cracks [23].

\section{Linear Elastic Interfacial Fracture Mechanics}

2.1 Oscillatory Singularity. The governing analytical solution for a plane interface crack between two elastic isotropic materials was obtained by Williams [20] and results in a singular, oscillating stress field as the crack tip is approached $(r \rightarrow 0$ in Fig. 1). The stress singularity is of the order of $r^{\xi}$, where $r$ is the distance from the crack tip, and $\xi=-1 / 2+i \varepsilon$ is a complex eigenvalue, where $i=\sqrt{-1}$ and $\varepsilon$ is the bimaterial constant defined as

$$
\varepsilon=\frac{1}{2 \pi} \ln \left[\frac{1-\beta}{1+\beta}\right]
$$

where

$$
\begin{gathered}
\alpha=\frac{\left[\bar{E}_{1}-\bar{E}_{2}\right]}{\left[\bar{E}_{1}+\bar{E}_{2}\right]} \\
\beta=\frac{\mu_{1}\left(\vartheta_{2}-1\right)-\mu_{2}\left(\vartheta_{1}-1\right)}{\mu_{1}\left(\vartheta_{2}+1\right)+\mu_{2}\left(\vartheta_{1}+1\right)}
\end{gathered}
$$

$\alpha, \beta$ are Dundur's parameters and $j=1,2$ represents material 1 and 2 (Fig. 1), respectively. Furthermore, $\vartheta_{j}=\left(3-4 \nu_{j}\right)$ and $\bar{E}_{j}=E_{j} /(1$ $\left.-\nu_{j}^{2}\right)$ for plane strain, and $\vartheta_{j}=\left(3-\nu_{j}\right) /\left(1+\nu_{j}\right)$ and $\bar{E}_{j}=E_{j}$ for plane stress where $E_{j}$ is the elastic modulus, $\nu_{j}$ is Poisson's ratio, and $\mu_{j}=E_{j} / 2\left(1+\nu_{j}\right)$ is the shear modulus, for material $j$.

Rice and Sih [21] introduced the complex stress intensity factor $\mathbf{K}$, defined by

$$
\mathbf{K}=K_{1}+i K_{2}=|\mathbf{K}| e^{i \Psi^{*}}
$$

where $\mathbf{K}$ has units of $\mathrm{Nm}^{-2} \sqrt{\mathrm{m}} \mathrm{m}^{-i \varepsilon}$ and $\Psi^{*}$ is the phase angle (though sometimes referred to as the mode mixity of $\mathbf{K}$, the defi- 


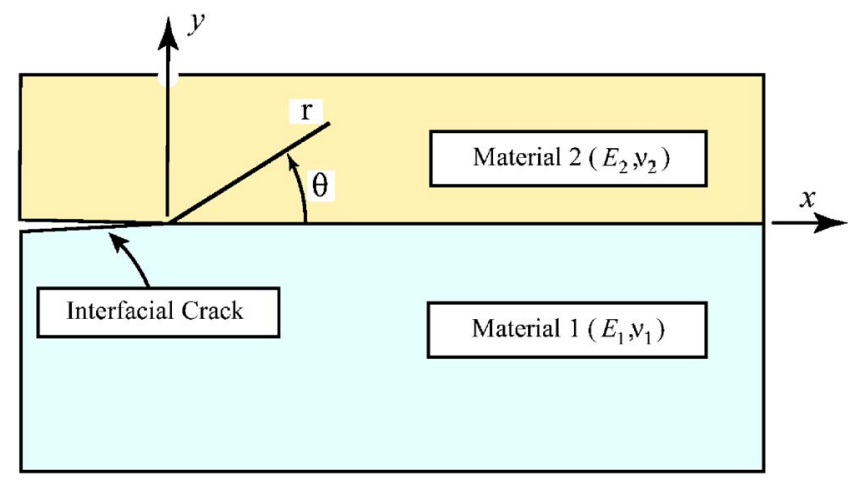

Fig. 1 Geometry and nomenclature of a bimaterial interface

nition of mode mixity used in this work is introduced in Sec. 2.2). $K_{1}$ and $K_{2}$ are scale sensitive and change with the dimensional units. The stress field ahead of the crack tip at the interface, $\theta$ $=0$, at a distance $r$ (Fig. 1) is given by [2,8,21]

$$
\sigma_{y y}+i \sigma_{x y}=\frac{\mathbf{K}}{\sqrt{2 \pi r}} r^{i \varepsilon}
$$

where $\sigma_{y y}$ is the tensile and $\sigma_{x y}$ the shear stress component.

The oscillatory solution implies: (i) coupled fracture modes [28], and (ii) interpenetration of crack faces adjacent to the crack tip [2]. Coupled fracture modes result in that $K_{1}$ and $K_{2}$ (Eq. (4)) cannot be interpreted as mode I and mode II stress intensity factors, similar to that of isotropic materials. Moreover, interpenetration of the crack faces is physically impossible, where experimental measurements of the near crack tip stress field through photoelasticity [31] and displacement field by moiré interferometry [32] indicate a nonoscillatory mode mixity condition near the crack tip. Thus, the oscillatory solution does not capture the behavior close to the crack tip correctly, for example, due to contact [29] and plasticity [9]. Several attempts have been made to improve the elastic solutions [20-23] containing the oscillating singularity, e.g., [29], even though these models are unable to determine the crack tip mode mixity [33,34]. Comninou's contact model assumes a small, nonlinear contact zone near the crack tip [29]. Although the contact model [29] is more realistic than the oscillatory solution, the analysis is cumbersome. In addition, the contact zone is much smaller than even the atomic dimensions for moderate values of mismatch $[11,23,27,35]$. The concept of a small-scale contact zone suggested by Rice [23] circumvents interpenetration of crack faces and allows the oscillatory solution to be valid in the $K$-annulus, i.e., the region close to the crack tip where the asymptotic singular field dominates, outside the nonlinear contact zone.

In spite of the limitations, linear fracture mechanics (including the oscillatory solution) is commonly used to determine the mode mixity of tested bimaterial systems [6-19,24-28], either analytically $[2,25]$ or through finite element simulation [26-28,30]. Thus, we will, regardless of the drawbacks of the oscillatory solution, use this approach due to its simplicity, by recasting Eq. (5) as discussed in the following.

2.2 Complex Stress Intensity Factors of Classical Type and Mode Mixity. Using the concept of reference length, an alternative definition of interfacial stress intensity factors (SIFs) was suggested by Rice [23], referred to as the complex stress intensity factors of classical type, which agrees with the definition of Malyshav and Salganik [22]:

$$
\sigma_{y y}+i \sigma_{x y}=\frac{K_{\mathrm{I}}+i K_{\mathrm{II}}}{\sqrt{2 \pi r}}\left(\frac{r}{l}\right)^{i \varepsilon}
$$

where $K_{\mathrm{I}}$ and $K_{\mathrm{II}}$ are complex SIFs of classical type based on a reference length $l$, where $l$ is an arbitrarily chosen distance $r=l$ ahead of the crack tip, where the stresses are measured. The stress intensity factors $K_{\mathrm{I}}$ and $K_{\mathrm{II}}$, Eq. (6), have the same units as the "isotropic" stress intensity factors, (i.e., $\mathrm{N} \mathrm{m}^{-2} \sqrt{\mathrm{m}}$ ), but have unclear physical meaning. For a bimaterial system with $\beta \neq 0, K_{\mathrm{I}}$ and $K_{\mathrm{II}}$ are not analogous to independent fracture modes I (opening) and II (in-plane shear) for homogeneous materials, since a reference length needs to be specified $[2,23]$.

The complex SIFs of classical type, $K_{\mathrm{I}}$ and $K_{\mathrm{II}}$, in Eq. (6) are related to the complex $K$ in Eq. (5) as

$$
K_{\mathrm{I}}+i K_{\mathrm{II}}=\mathbf{K} l^{i \varepsilon}=\mathbf{K} e^{i \psi}
$$

where $\psi$ is the mode mixity of $\mathbf{K} l^{i \varepsilon}$ and can be rewritten as

$$
\psi=\tan ^{-1}\left(\frac{K_{\mathrm{II}}}{K_{\mathrm{I}}}\right)=\tan ^{-1}\left(\frac{\operatorname{Im}\left(\mathbf{K} r^{i \varepsilon}\right)}{\operatorname{Re}\left(\mathbf{K} r^{i \varepsilon}\right)}\right)_{r=l}
$$

Furthermore, using Eq. (6), the mode mixity for an interface crack is expressed as

$$
\psi=\tan ^{-1}\left(\frac{\sigma_{x y}}{\sigma_{y y}}\right)_{r=l}
$$

where $\sigma_{y y}$ and $\sigma_{x y}$ are the stresses ahead of the crack tip at $l$. We note that the mode mixity defined by Eqs. (8) is a local mode mixity: the stresses and SIFs are local quantities, evaluated at a point defined by the reference length ahead of the crack tip. This local mode mixity is not the same as the global mode mixity, which is defined by the externally applied loads (see, for example, [23]). The global mode mixity depends on the crack length, i.e., a pure mode I external load can result in mixed mode conditions at the crack tip.

The selection of reference length $l$ in Eqs. (8) is arbitrary. Thus, $K_{\mathrm{I}}, K_{\mathrm{II}}$, and $\psi$ are arbitrary, since they depend on the reference length. From Eqs. (4) and (7), it follows that [2,6-9]:

$$
\psi={ }^{*}+\varepsilon \ln l
$$

leading to

$$
\psi_{2}=\psi_{1}+\varepsilon \ln \frac{l_{2}}{l_{1}}
$$

where $l_{1}$ and $l_{2}$ are two reference lengths used to define the mode mixities: $\psi_{1}=\psi_{1}\left(l_{1}\right)$ and $\psi_{2}=\psi_{2}\left(l_{2}\right)$. Thus, Eq. $(9 b)$ gives the relationship between two mode mixities and their associated reference lengths, for a given load condition.

The total strain energy release rate for an interface crack $G$ is not oscillatory and can be expressed as [2,22]

$$
G=\frac{1-\beta^{2}}{E^{*}} \mathbf{K}^{2}
$$

where

$$
\mathbf{K}^{2}=\left(K_{1}^{2}+K_{2}^{2}\right)=\left(K_{\mathrm{I}}^{2}+K_{\mathrm{II}}^{2}\right)
$$

and

$$
\frac{1}{E^{*}}=\frac{1}{2}\left(\frac{1}{\bar{E}_{1}}+\frac{1}{\bar{E}_{2}}\right)
$$

We note that $1-\beta^{2}=1 / \cosh ^{2}(\pi \varepsilon)$ and $l^{i \varepsilon}=1$. The strain energy release rate for an interface crack has the dimension of $\mathrm{Nm}^{-1}$ (which is the same as that for the strain energy release rate for monolithic material). At the fracture load, the critical total strain energy release rate of the interface $G_{C}$ is equal to the toughness of the interface $\Gamma_{\text {int }}$. 


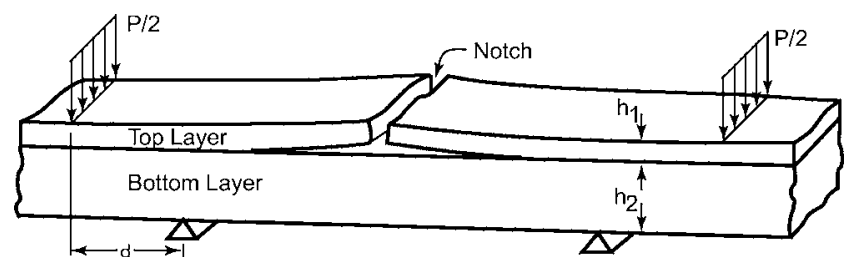

Fig. 2 Geometry and nomenclature of a notched, four-point flexure specimen

\section{Evaluation of Mode Mixity in Physical Test Speci- mens}

3.1 Aspects of a Material-Based Reference Length. As outlined above, the mode mixity of an interfacial crack must be specified with two parameters, mode mixity and reference length [2,23-28], and does not represent the conditions at the crack tip but at a distance $l$ in front of the crack tip. The reference length is based on an arbitrary selection, with no direct physical interpretation. It would be useful to define a "fixed" material based reference length $\hat{l}$, so to compare data from different specimens, e.g., $[2,7,23]$. Although the need for a reference length is an artifact of the mathematical solution, the "correct" value of $\hat{l}$, if known, can be used to uniquely and unambiguously represent the interfacial crack mode mixity in physical specimens.

A mode mixity based on a material-scale reference length $\hat{l}$ [23] is adopted by some authors $[2,7,10,12,15,16,23]$, even though there is no consensus on how to select $\hat{l}$ [2]. Since the fracture resistance of the interface is controlled by the shear and normal stresses in the fracture process zone, $\hat{l}$ could be based on a relevant microstructure dimension, such as grain size or the plastic zone [2,9,23,24]. Rice [23] suggested using $\hat{l}=1 \mu \mathrm{m}$, but emphasized that any other suitable value of $\hat{l}$ can be adopted. Cook and Erdogan [36] suggested the range $\hat{l}=2.54-254 \mu \mathrm{m} \quad(\hat{l}$ $=10^{-4}-10^{-2}$ in.). Alternatively, the selection of $\hat{l}$ is commonly based on a suitable choice outside the plastic zone $[7,9,11]$ or by judgment [12-16]. The mode mixity associated with interfacial toughness of the aluminum-epoxy system (which has properties similar to aluminum-vinyl ester system used in this study) has been based on various reference lengths, such as $\hat{l}=1 \mathrm{~mm}$ [12], $\hat{l}=100 \mu \mathrm{m}[11,15,18]$, and $\hat{l}=$ crack length [13]. Since the selection of such reference length is arbitrary, the mode mixity does not necessarily represent the conditions in the fracture process zone. Although failure criteria [7,12] can be adopted in terms of $K_{\mathrm{I}}$ and $K_{\mathrm{II}}$, they do not reveal the correct crack tip mode mixity condition controlling the interface fracture, as it depends on $\hat{l}$.

3.2 Characteristic Mode Mixity and Characteristic Reference Length. In this work, we propose and introduce a characteristic mode mixity $\psi_{c}$ based on the characteristic reference length $\hat{l}_{c}$, where $\hat{l}_{c}$ is assumed to be constant for a bimaterial pair. These two parameters are defined by (Eq. (8b))

$$
\psi_{c}=\tan ^{-1}\left(\frac{\sigma_{x y}}{\sigma_{y y}}\right)_{r=\hat{l}_{c}}
$$

where $\sigma_{y y}$ and $\sigma_{x y}$ are the stresses ahead of the crack tip at $\hat{l}_{c}$. In the following, we outline how the characteristic reference length and the characteristic mode mixity can be established for a given material combination using interface toughness data and linear elastic interfacial mechanics.

Consider two sets of specimens, $A$ and $B$, of the same bimaterial system. (For example, in the experimental work discussed in Sec. 4, we use a notched four-point bend test, Figs. 2 and 3, with

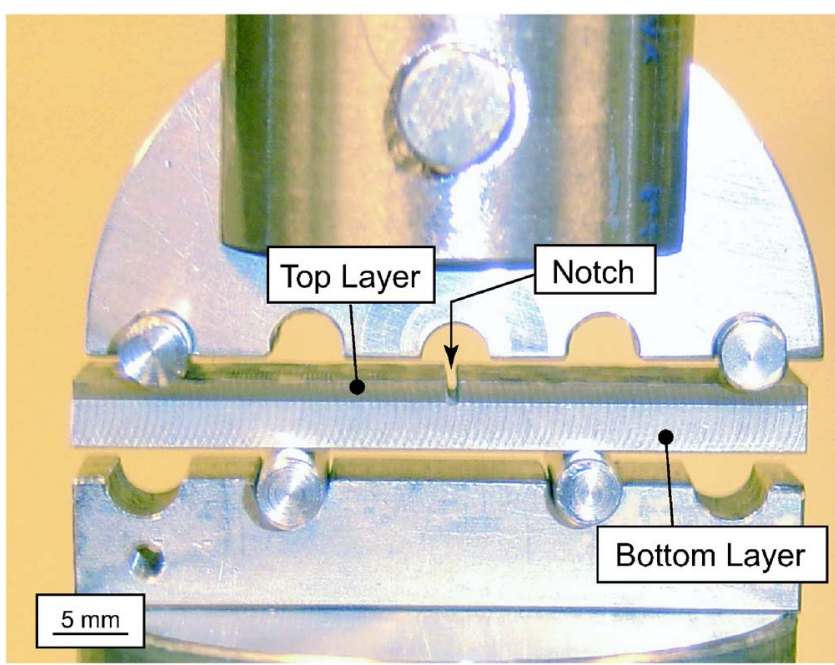

Fig. 3 Experimental setup

the bimaterial samples made from aluminum and vinyl ester. In set $A$, the vinyl ester is the notched top layer with aluminum as the intact bottom layer. Set $B$ is the reversed case, the aluminum as the notched top layer and vinyl ester as the intact bottom layer.) Assuming linear-elastic interfacial mechanics, the mode mixity based on the reference length $l_{1}$, i.e., $\psi_{1}=\psi\left(l_{1}\right)$, can be related to another mode mixity based on the reference length $l_{2}$, i.e., $\psi_{2}$ $=\psi\left(l_{2}\right)$, using Eq. $(9 b)$, according to the following:

$$
\begin{aligned}
& \left(\psi_{2}\right)_{A}=\left(\psi_{1}\right)_{A}+\varepsilon_{A} \ln \frac{\left(l_{2}\right)_{A}}{\left(l_{1}\right)_{A}} \\
& \left(\psi_{2}\right)_{B}=\left(\psi_{1}\right)_{B}+\varepsilon_{A} \ln \frac{\left(l_{2}\right)_{B}}{\left(l_{1}\right)_{B}}
\end{aligned}
$$

where $\varepsilon_{\mathrm{A}}$ and $\varepsilon_{\mathrm{B}}$ are the bimaterial parameters for specimen sets $A$ and $B$, Eq. (1).

Since specimen sets $A$ and $B$ are of the same bimaterial system, the properties of the interface, thus the toughness curve (interfacial fracture toughness as a function of mode mixity), in the two sets of specimens should be identical. ${ }^{2}$ Furthermore, assuming that the specimen sets $A$ and $B$ have interchanged layers of the two materials, the bimaterial constant $\varepsilon$ of specimens $A$ and $B$ have opposite signs but the same magnitude, i.e., $\varepsilon_{A}=-\varepsilon_{B}=\varepsilon$. In Eq. ( $8 b)$ and, thus, in Eq. (12), $l_{1}$ and $l_{2}$ can be arbitrarily chosen and need not necessarily be in the $k$-annulus region. We choose identical reference lengths for specimen sets $A$ and $B$, i.e., $\left(l_{1}\right)_{A}$ $=\left(l_{1}\right)_{B}=l_{1}$, and $\left(l_{2}\right)_{A}=\left(l_{2}\right)_{B}=l_{2}$. Furthermore, let $l_{2}$ equal the characteristic reference length, $l_{2}=\hat{l}_{c}$. Then, Eq. (12) can be rewritten as

$$
\begin{aligned}
& \left(\psi_{c}\right)_{A}=\left(\psi_{1}\right)_{A}+\varepsilon \ln \left(\frac{\hat{l}_{c}}{l_{1}}\right) \\
& \left(\psi_{c}\right)_{B}=\left(\psi_{1}\right)_{B}-\varepsilon \ln \left(\frac{\hat{l}_{c}}{l_{1}}\right)
\end{aligned}
$$

In Eq. (13), $l_{1}$ is a convenient length, used to determine the mode mixities $\left(\psi_{1}\right)_{A}$ and $\left(\psi_{1}\right)_{B}$ from a finite element simulation or from an analytical solutions with fracture load from the physical test. The characteristic reference length $\hat{l}_{c}$ and the mode mixities, $\left(\psi_{c}\right)_{A}$ and $\left(\psi_{c}\right)_{B}$, are unknowns, determined by using additional

${ }^{2}$ Some similarities between the specimens must be assumed, e.g., both sets can be considered as either plane strain or plane stress. 
Table 1 Dimensions of the specimens

\begin{tabular}{|c|c|c|c|c|c|}
\hline \multirow[b]{2}{*}{ Set No. } & \multicolumn{5}{|c|}{$\begin{array}{l}\text { Specimen } A, \varepsilon_{A}=0.0995 \\
\text { Top layer: vinyl ester }\end{array}$} \\
\hline & $\begin{array}{l}\text { Specimen } \\
\text { No. }\end{array}$ & $h_{1}$ & $h_{2}$ & $\eta=h_{1} / h_{2}$ & $\begin{array}{c}\text { Number of } \\
\text { samples }\end{array}$ \\
\hline 1 & $A 1$ & 2.28 & 4.75 & 0.48 & 6 \\
\hline 2 & $A 2$ & 3.03 & 3.13 & 0.97 & 6 \\
\hline 3 & $A 3$ & 1.61 & 3.13 & 0.51 & 4 \\
\hline 4 & $A 4$ & 2.57 & 1.93 & 1.33 & 4 \\
\hline \multirow[t]{2}{*}{5} & $A 5$ & 1.11 & 1.93 & 0.58 & 5 \\
\hline & \multicolumn{5}{|c|}{$\begin{array}{l}\text { Specimen } B, \varepsilon_{B}=-0.0995 \\
\text { Top layer: aluminum }\end{array}$} \\
\hline Set No. & $\begin{array}{l}\text { Specimen } \\
\text { No. }\end{array}$ & $h_{1}$ & $h_{2}$ & $\eta=h_{1} / h_{2}$ & $\begin{array}{c}\text { Number of } \\
\text { samples }\end{array}$ \\
\hline 6 & $B 1$ & 1.93 & 2.57 & 0.75 & 5 \\
\hline 7 & $B 2$ & 0.93 & 2.75 & 0.34 & 4 \\
\hline 8 & B3 & 0.93 & 1.62 & 0.57 & 6 \\
\hline 9 & $B 4$ & 0.77 & 3.08 & 0.25 & 1 \\
\hline
\end{tabular}

information from the interfacial toughness curve. Since the toughness curve is a property of the bimaterial interface, the fracture toughness data of specimen sets $A$ and $B$ should be reproducible, independent of the specimen type (it is assumed that the toughness variation due to the specimen size, on account of $K$-annulus effects [35], are negligible). Thus, the toughness curves for a given bimaterial system, obtained from various types of test specimens, can be combined if the mode mixity, is based on the characteristic reference length, i.e., the characteristic mode mixity.

For some toughness value, the toughness of specimens $A$ and $B$ must be equal. We denote the point of equal toughness as the intersection point, i.e., point $A^{\oplus}$ on the toughness curve for specimen $A$ has same toughness as point $B^{\oplus}$ on the toughness curve for specimen $B$ :

$$
\left(\Gamma_{\text {int }}\right)_{A^{\otimes}}=\left(\Gamma_{\text {int }}\right)_{B^{\otimes}}
$$

The intersection point occurs for a particular combination of loading and specimen geometry. The mode mixities at this point, $\left(\psi_{1}\right)_{A^{\otimes}}$ and $\left(\psi_{1}\right)_{B^{\otimes}}$, are both based on the reference length $l_{1}$. Now, can we select a reference length so that the mode mixities at the intersection point are equal? Yes, we can, and this is the characteristic reference length, $\hat{l}_{c}$, which is associated with the characteristic mode mixity, $\psi_{c}$. Thus, at the intersect point

$$
\left(\psi_{c}\right)_{A^{\otimes}}=\left(\psi_{c}\right)_{B^{\otimes}}=\left(\psi_{c}\right)^{\otimes}
$$

Consequently, Eq. (13) should be written as

$$
\begin{aligned}
& \left(\psi_{c}\right)_{A^{\otimes}}=\left(\psi_{1}\right)_{A}+\varepsilon \ln \left(\frac{\hat{l}_{c}}{l_{1}}\right) \\
& \left(\psi_{c}\right)_{B^{\otimes}}=\left(\psi_{1}\right)_{B}-\varepsilon \ln \left(\frac{\hat{l}_{c}}{l_{1}}\right)
\end{aligned}
$$

It follows that from Eqs. (14b) and (15), we get

$$
\left(\psi_{1}\right)_{B^{\otimes}}=\left(\psi_{1}\right)_{A^{\otimes}}+2 \varepsilon \ln \left(\frac{\hat{l}_{c}}{l_{1}}\right)
$$

Thus, we can determine $\hat{l}_{c}$ from Eq. (16) and $\left(\psi_{c}\right)^{\otimes}$ is then obtained from Eqs. (14b) and (15). As will be illustrated in Sec. 5, the intersection points for specimen sets $A$ and $B$ can be determined from experimental fracture data based on a number of specimens of sets $A$ and $B$.
Table 2 Material properties

\begin{tabular}{lcc}
\hline \hline Material & $E$ & \\
\hline Vinyl ester & $(\mathrm{GPa})$ & $\nu$ \\
Aluminum & 2.48 & 0.35 \\
\hline \hline
\end{tabular}

\section{Experiments Using Bilayer Four-Point Flexure Specimen}

4.1 Test Methodology. The concept developed above will be elucidated by experimental investigations. Bilayer four-point flexure specimens with a center notch (Fig. 2) ("mixed-mode delaminating beam" (MMDB) specimen, or UCSB specimen), originally designed by Evans et al. [1] and Charalambidies et al. [6], will be employed. This specimen has been used extensively for interfacial testing of bimaterial interfaces, composite laminates, metallic adhesive joints, and thin films $[2,3,5,6,8,19,25,37]$. The specimen contains a notch in the center of the top layer, which reaches the interface (Fig. 2). On loading in four-point flexure, interface cracks grow on both sides of the notch at the fracture load. The specimen has a simple fixture and an advantageous configuration such that when the interface crack extends a few times the thickness of the top layer, it can be considered as semi-infinite and undergoes steady-state propagation [6]. Between the inner loading points, the bending moment is constant. Thus, the mode mixity remains constant as the crack propagates and it is not necessary to monitor the crack length accurately.

4.2 Specimens. We investigate the interfacial fracture resistance of an aluminum and vinyl ester bimaterial system using two sets of bilayer four-point flexure specimens: specimen $A$ and specimen $B$. Specimen $A$ has vinyl ester as the top layer containing a notch in the center, and the bottom layer is aluminum. Specimen $B$ is the reverse of $A$ : it has aluminum as the top layer with a center notch and vinyl ester as the bottom layer. The thickness of the top layer is $h_{1}$, and that of the bottom layer is $h_{2}$. Thus, the total thickness of specimen is $h=h_{1}+h_{2}$. The length and width of all the specimens are $L=50.8 \mathrm{~mm}$ and $b=12.7 \mathrm{~mm}$, respectively. The distance between the inner support pins is $20 \mathrm{~mm}$ and between outer loading pins is $40 \mathrm{~mm}$. Thus, the distance between loading pins and support pins on both sides of the notch is $d$ $=10 \mathrm{~mm}$.

Various combinations of $h_{1}$ and $h_{2}$ are considered for each set of specimens $A$ and $B$, as shown in Table 1; thus, to obtain various mode mixities $[2,25]$. The mechanical properties of the two materials are shown in Table 2 . The modulus of elasticity for vinyl ester is determined from a three-point bend test. Vinyl ester is a brittle material with linear-elastic behavior. The aluminum is assumed to respond linear elastically during the test. Poisson's ratio $\nu$ of the two materials is based on the published data $[11,38]$.

Plane strain condition is assumed. Thus, for this bimaterial system from Eqs. (1)-(3), we have $\alpha=-0.9318, \beta=-0.3028$, and $\varepsilon_{A}=0.0995$ for specimen $A$, and $\alpha=-0.9318, \beta=-0.3028$, and $\varepsilon_{B}=-0.0995$ for specimen $B$.

4.3 Sample Preparation. The bilayer test specimens (sets $A$ and $B$ ) are prepared by using aluminum 6061-T6 and a commercial vinyl ester resin (Derakane $\left.{ }^{\circledR} 411-350\right){ }^{3}$ The surface of the aluminum plate is sandblasted to increase the adhesion and then cleaned with acetone, prior to casting the resin. Using $0.2 \mathrm{wt} \%$ cobalt naphthenate as catalyst (accelerator) and $1 \mathrm{wt} \%$ organic peroxide (Trigonox $\left.{ }^{\circledR} 239 \mathrm{~A}\right)^{4}$ as the initiator, liquid vinyl ester resin is cast into molds with aluminum as the base plate. All

\footnotetext{
${ }^{3}$ Derakane ${ }^{\circledR}$ is a registered trademark of Ashland Specialty Chemicals Co.
}

${ }^{4}$ Trigonox ${ }^{\circledR}$ is registered trademark of Akzo Nobel Polymer Chemicals LLC. 
Table 3 Fracture load

\begin{tabular}{|c|c|c|c|c|c|c|}
\hline \multirow[b]{2}{*}{$\begin{array}{l}\text { Specimen } \\
\text { No. }\end{array}$} & \multicolumn{6}{|c|}{$\begin{array}{l}\text { Fracture load of samples per unit width } P / b \\
\qquad(\mathrm{~N} / \mathrm{mm})\end{array}$} \\
\hline & 1 & 2 & 3 & 4 & 5 & 6 \\
\hline$A 1$ & 84.25 & 85.04 & 93.99 & 86.61 & 82.21 & 82.34 \\
\hline$A 2$ & 24.96 & 25.95 & 26.46 & 26.30 & 22.75 & 27.72 \\
\hline$A 3$ & 45.50 & 44.79 & 51.97 & 47.87 & & \\
\hline A4 & 13.11 & 10.90 & 11.93 & 10.82 & & \\
\hline$A 5$ & 20.03 & 21.04 & 24.84 & 22.52 & 21.97 & \\
\hline$B 1$ & 3.50 & 3.41 & 3.26 & 3.58 & 3.89 & \\
\hline$B 2$ & 4.68 & 5.40 & 5.01 & 4.68 & & \\
\hline$B 3$ & 1.88 & 2.28 & 2.28 & 2.12 & 2.35 & 2.20 \\
\hline$B 4$ & 7.26 & & & & & \\
\hline
\end{tabular}

specimens are cured at room temperature for $72 \mathrm{~h}$ and then milled to the specified dimensions under identical conditions. Subsequently, for the specimens of set $A$, a straight-through vertical notch (Fig. 2) is made in the center of vinyl ester layer by a blade (0.4 mm thickness). For specimens of set $B$, the notch is made in the center of aluminum layer in a similar manner. One to six samples are used for each combination of layer thicknesses, $h_{1}$ and $h_{2}$ for specimen sets $A$ and $B$ in Table 1 .

4.4 Interfacial Toughness Testing. The bilayer specimens are tested in a four-point flexure fixture (Fig. 3) using a MTS 856 hydraulic strength test machine under displacement controlled condition at constant rate of $0.025 \mathrm{~mm} / \mathrm{min}$. The friction between specimen and loading pins is minimized by using a Teflon ${ }^{\circledR}$ tape. Although precracking of the interface crack may eliminate errors due to notch effects, no precracking is introduced in the specimens because it is difficult to control the length of the interfacial precracks (e.g., [39]).

The interface fracture is observed visually with the aid of an optical microscope. For the specimens with the thicker top layer, the expected force plateau (nonlinearity) on the forcedisplacement curve (corresponding to the steady-state region $[3,6,40]$ after interface crack initiation) cannot be distinguished. Hence, the fracture load is assumed to correspond to the initiation of the interface crack from the notch. The largest source of scatter in the fracture load is due to misalignment of the loading pins resulting in that the interfacial crack do not initiate simultaneously at the two sides of the notch. For simplicity, no special arrangement [41] to correct the error due to asymmetric crack advance was used. Therefore, the samples with more than $10 \%$ difference in the fracture load for crack initiation from the two sides of the notch were discarded. For smaller variations, the average of the two values was assumed as the fracture load of the sample, summarized in Table 3. The scatter in the experimental data is comparable to that available in the literature $[8,11,13-16,19]$. However, the scatter could be reduced by implementing precracks and utilize a mounting fixture to assure proper alignment of the specimens, as discussed above.

4.5 Specimen Analysis. Assuming steady-state condition and no residual stresses, the total strain energy release rate can be determined analytically as the difference in the elastic strain energy in the cracked and uncracked parts of the four-point flexure specimen (Fig. 2). The strain energy of the top layer in the cracked part of the beam is neglected. Using Euler-Bernoulli beam theory with plane strain condition, the total energy release rate $G$, Eq. $(10 a)$, is [6]

\footnotetext{
${ }^{5}$ Teflon $^{\circledR}$ is a registered trademark of DuPont Company.
}

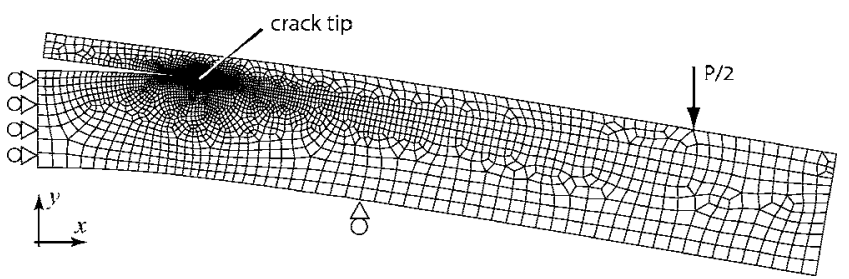

Fig. 4 Finite element model of the four-point flexure specimen where boundary conditions and imposed loads are indicated (shown under loading with exaggerated deformations)

$$
G=\frac{M^{2}}{2 E_{2}}\left(\frac{1}{I_{2}}-\frac{\rho}{I_{c}}\right)
$$

where $M=P d / 2 b(\mathrm{Nmm} / \mathrm{mm})$ is the net bending moment per unit width, and $P$ is the applied force per unit width $(\mathrm{N} / \mathrm{mm})$. Furthermore, $\rho=\bar{E}_{2} / \bar{E}_{1}$ is the ratio of effective modulus, $I$ denotes the area moment of inertia per unit width, subscripts 2 refers to the bottom layer, and $c$ refers to the equivalent composite beam. At fracture load, the total strain energy release rate in Eq. (17) is denoted $G_{C}$ and equals the interfacial toughness $\Gamma_{\text {int }}$.

However, residual stresses are often present in multilayered structures $[3,16,42,43]$. In contrast to thin-layer sandwich specimens $[3,10,11,13]$, the residual stresses generally contribute to the crack driving force in the four-point flexure specimen and can significantly influence the values of strain energy release rate and mode mixity $[8,42]$. For the aluminum-vinyl ester bilayer fourpoint flexure specimens, residual stresses occur due to shrinkage of the resin during curing. The residual stress is incorporated as an equivalent thermal misfit strain [42], described in the Appendix A, when evaluating in the four-point flexure specimens. This necessitates the use of finite element analysis, incorporated as follows.

The fracture parameters (the toughness and the mode mixity) for the test specimens are determined based on finite element analysis of the four-point flexure specimen using the commercially available program ABAQUS [44]. A two-dimensional finite element model is used assuming the plane strain condition using four-node linear quadrilateral elements, adapted from previous work [27]. Symmetry about the $y$-axis at the midsection of the specimen is imposed to reduce the model size (Fig. 4). Elastic material properties of aluminum and vinyl ester (Table 2) are used. For a linear-elastic analysis, the total energy release rate and the mode mixity (computed numerically) agrees with the analytical results, i.e., Eq. (17) in the absence of residual stresses $[2,25,27]$.

In the finite element model, mechanical force corresponding to the fracture load (Table 3), and a uniform thermal misfit strain (Appendix A) to simulate the residual stresses are applied. The deformed shape of the model is shown in Fig. 4 on an exaggerated scale. The total strain energy release rate for the above loading, $G_{C}$, corresponds to the interface toughness of the sample, $\Gamma_{\text {int }}$.

The mode mixity is computed from the values of the real and imaginary parts of the complex stress intensity factors, $K_{1}$ and $K_{2}$, Eq. (4). These values are available directly from ABAQUS [44]. From Eq. (9a), it follows that for a reference length $l$ of unit length (in this case, millimeters), * , defined by Eq. (4), equals the mode mixity $\psi$, defined by Eq. (7). Thus, for simplicity we select to use reference length $l_{1}=1 \mathrm{~mm}$. The applicability of Eq. $(9 a)$, along with the validity and accuracy of the results from ABAQUS [44], has been compared to other approaches available in the literature through various numerical examples in our previous study using a modified virtual crack closure technique [27], not shown for brevity. Thus, the mode mixity values obtained from the finite element analysis refer to $\left(\psi_{1}\right)_{A}$ and $\left(\psi_{1}\right)_{B}$ (Eq. (15)) for the specimen sets $A$ and $B$, corresponding to the reference length, $l_{1}=1 \mathrm{~mm}$. The effect of friction at the loading pins is small for a 

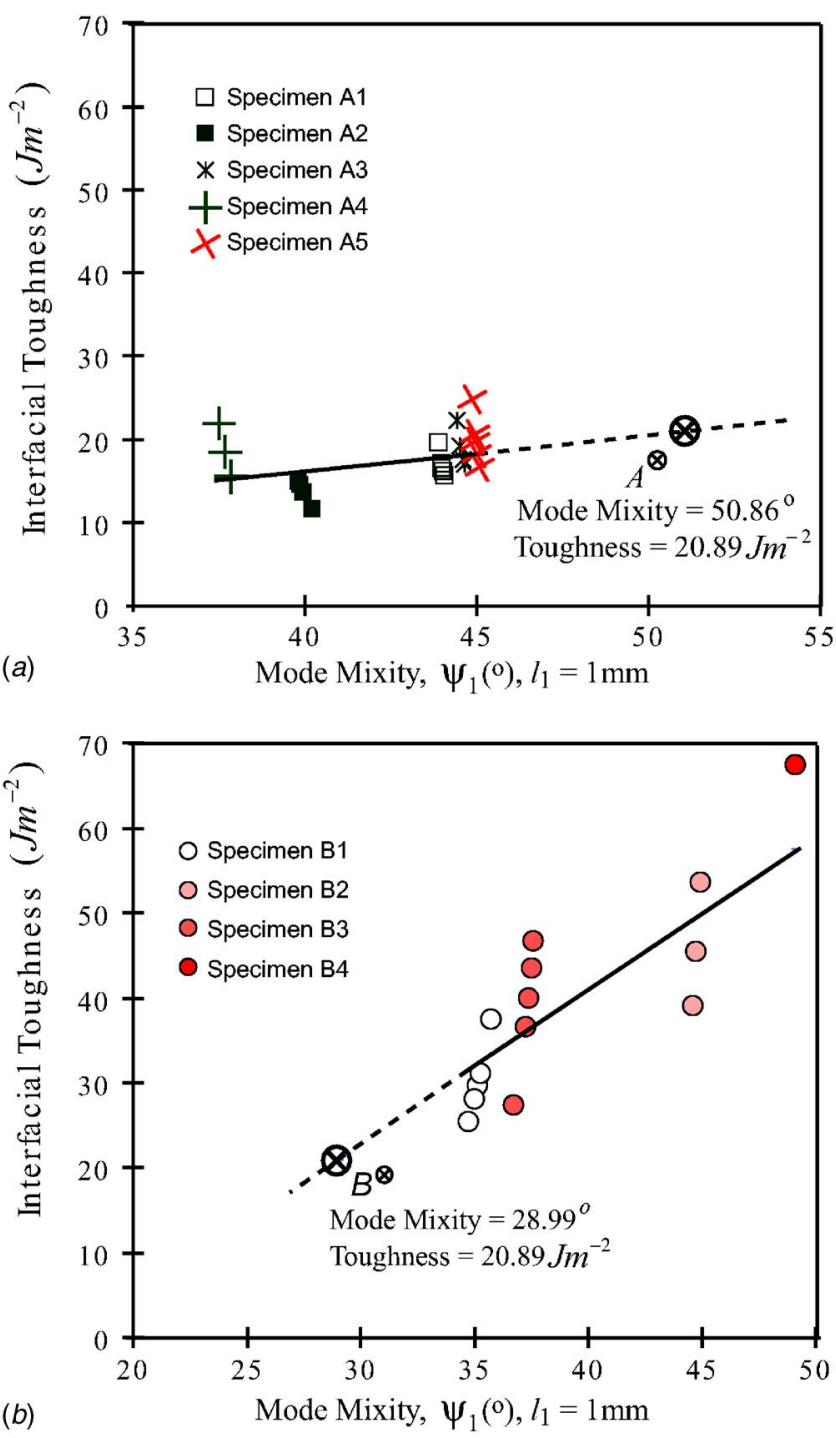

Fig. 5 Interface toughness curve for specimen sets $A$ and $B$

large $d / h$ ratio [42] and is neglected in the present study.

\section{Results}

For each sample, the interfacial toughness and the associated mode mixity $\psi_{1}$ (based on reference length $l_{1}=1 \mathrm{~mm}$ ) is determined from finite element results using the fracture load (and the uniform thermal misfit strain). The toughness of set $A$ ranges from $15 \mathrm{Jm}^{-2}$ to $18 \mathrm{Jm}^{-2}$, whereas for set $B$ specimens, the toughness ranges from $31 \mathrm{Jm}^{-2}$ to $58 \mathrm{Jm}^{-2}$ (Fig. 5). The mode mixity (based on reference length $l_{1}=1 \mathrm{~mm}$ ) varies within a range of about $8 \mathrm{deg}$ for set $A$ and $14 \mathrm{deg}$ for set $B$ (Fig. 5). A linear fit of the interfacial toughness curve is assumed due to the scatter of the data. (The scatter of the interface toughness is enhanced from the experimental data since $G_{C}$, thus $\Gamma_{\text {int }}$, is proportional to the square of the fracture load, Eq. (17).)

The intersection point (denoted $\otimes$ ), corresponding to the point of equal toughness, is determined by extrapolating the toughness curves of specimens $A$ and $B$. As discussed earlier, higher mode mixities (more shear stress contribution) should correspond to higher interfacial fracture toughness. Specimen $B$ results in the higher fracture toughness (Fig. 5) and should therefore correspond to the higher range of the characteristic mode mixity. Thus, the toughness curve obtained from set $A$ should be extrapolated toward higher mode mixities and the toughness curve from set $B$

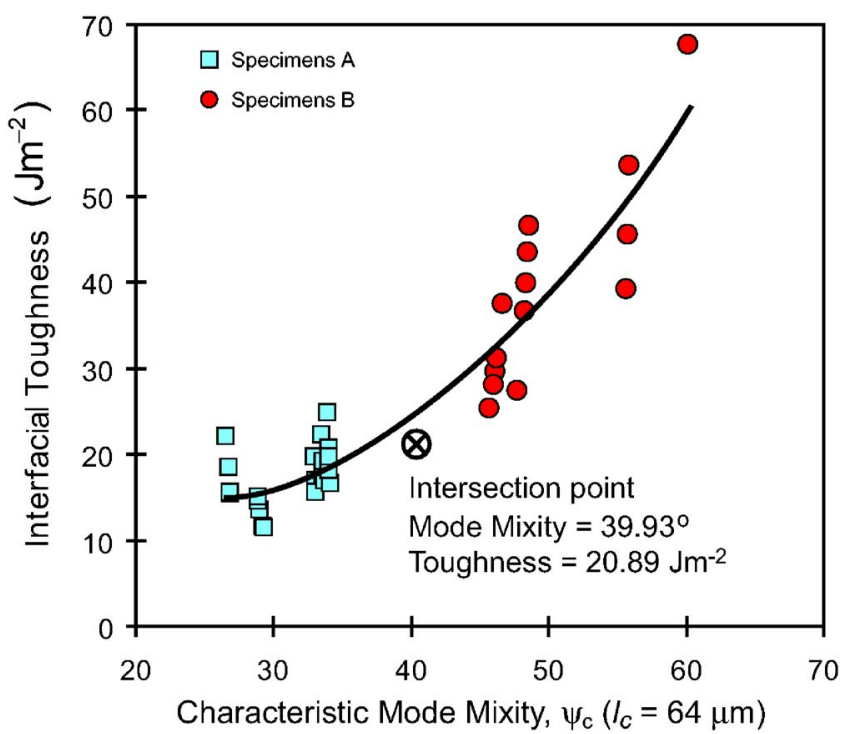

Fig. 6 Combined interface toughness curve

should be extrapolated toward lower mode mixities, as indicated by the dotted lines in Fig. 5. The intersection point is selected so that equal amount of shift in $\psi_{1}$ on the two toughness curves is made. ${ }^{6}$ In this case, the toughness curve for set $A$ specimens is extrapolated by increasing $\psi_{A}$ with $10.95 \mathrm{deg}$, and decreasing $\psi_{B}$ by the same amount. Thus, the intersection point has in interfacial toughness of $20.89 \mathrm{Jm}^{-2}$, corresponding to mode mixity $\left(\psi_{1}\right)_{A}$ $=50.86 \mathrm{deg}\left(l_{1}=1 \mathrm{~mm}\right)$ and $\left(\psi_{1}\right)_{B}=28.99 \mathrm{deg}\left(l_{1}=1 \mathrm{~mm}\right)$. From Eq. (16), the characteristic reference length associated with this particular material system is determined to be $\hat{l}_{c}=64 \mu \mathrm{m}$ and the intersecting mode mixity is obtained from Eqs. (14b) and (15), determined to be $\left(\psi_{c}\right)^{\otimes}=\left(\psi_{c}\right)_{A^{\otimes}}=\left(\psi_{c}\right)_{B^{\otimes}}=39.93 \mathrm{deg}$.

We can now combine the toughness curves for the aluminumvinyl ester bimaterial specimens into one curve. The combined curve is the interfacial fracture toughness as a function of the characteristic mode mixity $\psi_{c}$, which is based on the characteristic reference length $\hat{l}_{c}=64 \mu \mathrm{m}$, Fig. 6 . The combined toughness curve shows that the characteristic mode mixity obtained from the two sets of four-point flexure specimens covers a relatively larger range (from about $27 \mathrm{deg}$ to $60 \mathrm{deg}$ ) for the aluminum-vinyl ester bimaterial pair.

According the Eq. (9b), the approach is insensitive to the initial selection of the arbitrary reference length $l_{1}$; this equation relates the mode mixity for one reference length with mode mixity associated with another reference length. We redid the above calculations when assuming an initial arbitrary reference length of $l_{1}$ $=0.5 \mathrm{~mm}$, obtaining the same characteristic reference length (not shown for brevity).

As a final note on these results, we would like to point out that there was significant scatter in the experimental data. Thus, the characteristic reference length $\hat{l}_{c}=64 \mu \mathrm{m}$ should be used with care. For example, due to the scatter, we assumed a linear extrapolation of the results presented in Fig. 5, even though it may be expected that the toughness curve is of parabolic shape, e.g., [2]. By using the standard deviation of the experimental data to establish upper and lower bounds of the interfacial toughness curves in Fig. 5 and redoing the analysis shown above, the characteristic reference length is $\hat{l}_{c}=64 \pm 6 \mu \mathrm{m}$.

\footnotetext{
${ }^{6}$ There are infinitely many possibilities to select the intersection point, since the range of the $\psi$-axis is not the same for $A$ and $B$, and we only match the $\Gamma_{\text {int }}$-axis. The seemingly arbitrary approach used here would converge to a specific value if enough tests were done so that the extrapolation would not be needed.
} 

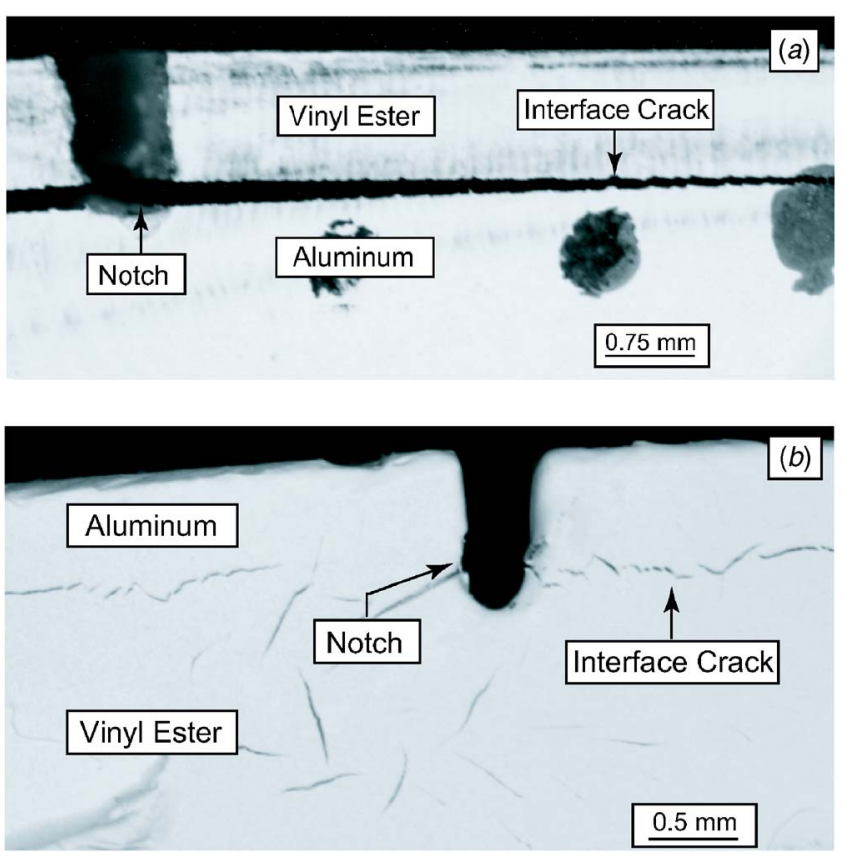

Fig. 7 Fracture pattern for (a) specimens of type $A 1$ and (b) specimens of type $B 4$

\section{Discussion}

6.1 Interfacial Fracture Patterns. The fracture pattern for the interfacial crack propagation is observed in situ by an optical microscope for each sample. For all $A$ specimens, the fracture is similar to the cleavage fracture associated with mode I in homogeneous materials, whereas for the $B$ specimens the fracture is jagged, showing the attributes of mode II fracture in homogeneous materials. This agrees with the characteristic mode mixity in the combined toughness plot, where the $A$ specimens have low mode mixity and the $B$ specimens have higher characteristic mode mixity (Fig. 7).

6.2 Stress Intensity Factors Based on the Characteristic Mode Mixity. The characteristic mode mixity $\psi_{c}$, based on the characteristic reference length $\hat{l}_{c}$, is defined by Eq. (11). Hence, once $\hat{l}_{c}$ is established for a bimaterial pair, the ambiguity arising due to oscillatory singularity can be circumvented. Based on the characteristic mode mixity $\psi_{c}$, the characteristic complex stress intensity factors of classical type, $K_{\mathrm{I}}^{c}$ and $K_{\mathrm{II}}^{c}$, can be regarded as effective mode I and mode II corresponding to SIFs of classical type, similar to homogeneous, isotropic materials $[12,45]$. To analyze fracture, $K_{\mathrm{I}}^{c}$ and $K_{\mathrm{II}}^{c}$ need to be compared with the $\left(K_{\mathrm{I}}^{c}\right)_{\text {crit }}$ and $\left(K_{\text {II }}^{c}\right)_{\text {crit }}$, which are the effective mode I and mode II fracture toughnesses of the bimaterial interface based on $\hat{l}_{c}$. The mixed mode interface fracture can be analyzed as [12]

$$
\left(\frac{K_{\mathrm{I}}^{c}}{\left(K_{\mathrm{I}}^{c}\right)_{\text {crit }}}\right)^{2}+\left(\frac{K_{\mathrm{II}}^{c}}{\left(K_{\mathrm{II}}^{c}\right)_{\text {crit }}}\right)^{2}=1
$$

Thus, the criteria for interfacial fracture can be established similar to that for the isotropic homogeneous materials when based on the characteristic mode mixity.

6.3 Comparison of the Results for the Characteristic Mode Mixity. To our knowledge, there are no other methods published in the open literature on how to establish a materialbased reference length $\hat{l}$. In fact, mode mixity is obtained analytically or numerically using the oscillatory solution with an arbitrary reference length for most experimental studies, e.g., [8-18].
Thus, a direct comparison of the characteristic mode mixity, obtained in this work, based on $\hat{l}_{c}$, is not possible. However, a few suggested values of a materials-based reference length are available. For example, after obtaining the toughness curve, the $\psi$-axis can be shifted by selecting various reference lengths so that the minimum toughness corresponds to the zero mode mixity $[2,9,12,14,16]$. Based on this approach, Ikeda et al. [12] suggested $\hat{l}=10 \mu \mathrm{m}$, whereas Wang and Suo [11] used $\hat{l}=100 \mu \mathrm{m}$ for aluminum epoxy. The possibility of linking $\hat{l}$ to Dundurs parameters has been suggested [14], but specific values are not available in the literature. Alternatively, $\hat{l}$ can be established based on the plastic zone size. Based on literature values [46], the yield strength for vinyl ester is $62 \mathrm{MPa}$. We find that the plastic zone size varies from $\sim 8 \mu \mathrm{m}$ for the specimens of type $A$ up to $\sim 60 \mu \mathrm{m}$ for specimens of type $B$. Thus, the plastic zone size gives little insight into the selecting an appropriate materials-based reference length.

\section{Concluding Remarks}

We have investigated properties that govern interfacial fracture: interfacial fracture toughness, mode mixity, and the associated reference length, based on linear-elastic fracture mechanics. For a bimaterial interface crack, mode mixity is not as clearly defined as it is for isotropic materials, due to the oscillatory solution obtained near the crack tip. Thus, an additional parameter, the "reference length" is needed to characterize interfacial fracture. The reference length describes the location at where the mode mixity is evaluated ahead of the crack tip. There are no fixed guidelines on how to select the reference length. Thus, we investigate and propose an experimental method on how to establish a materials based reference length, referred to as the "characteristic reference length," and the associated mode mixity, referred to as the "characteristic mode mixity," within the context of linear fracture mechanics.

The proposed method is based on the postulate that if two specimens are of the same bimaterial system, the properties of the interface of the two specimens are identical. To elucidate the proposed method, the toughness curve (i.e., the interfacial fracture toughness as a function of the mode mixity) of a bimaterial system (aluminum and vinyl ester) is obtained by using two sets of the four-point flexure specimens. The specimens are designed to cover a broad range of mode mixities. The procedure proposed is detailed in the paper and can be summarized by

1. Test two sets of specimens to failure.

2. For each sample tested, use finite element simulations to establish the corresponding interfacial fracture toughness as a function of the mode mixity, based on one arbitrary (but fixed) reference length.

3. Determine an intersection point where the interfacial fracture toughness is equal for the two cases.

4. For that point, use the mathematical relationship that translates mode mixities based on one reference length to another and determine a reference length and mode mixity so that the toughness curve for the two types of specimens become continuous. This is the characteristic reference length $\hat{l}_{c}$ and the associated characteristic mode mixity $\psi_{c}$. For the bimaterial system investigated (aluminum and vinyl ester) the characteristic length was determined to be $64 \mu \mathrm{m}$ with a standard deviation of $\sim 10 \%$.

The current work does not give any additional insight into whether the characteristic reference length corresponds to any physical quantities, but this is currently being investigated. We believe that our proposed method serves as a useful benchmark method to estimate experimentally meaningful values for the characteristic reference length, which can serve as an important fracture parameter. 


\section{Appendix: Analysis of Residual Stress}

Shrinkage of $0.2 \%$ in the vinyl ester resin occurs during curing and leads to biaxial tension. For a two-dimensional case, a uniform misfit stress $[25,42,43], \sigma^{R}$ can be estimated as

$$
\sigma^{R}=\bar{E} \Theta
$$

where $\bar{E}=E$ for plane stress and $\bar{E}=E /\left(1-\nu^{2}\right)$ for plane strain. $E$ and $\nu$ are the elastic modulus and Poisson's ratio, respectively, of vinyl ester, and $\Theta$ is the effective shrinkage strain in the vinyl ester. However, since the shrinkage occurs during curing, the elastic modulus listed in Table 2 is not reached until the end of the curing/shrinkage. Thus, an ad hoc approach is used to estimate the residual stress. Averaging over the curing time gives average uniform misfit strain as $0.1 \%$. In addition, $E_{v e}$ could be assumed as one-fourth of the value in Table 2 because of initial liquid state. Using these values in Eq. (A1), we get the misfit stress $\sigma^{R}$ $=0.837 \mathrm{MPa}$. Using value of $E_{v e}$, from Table 2, the effective misfit strain is $\Theta=0.25 \%$. This is applied as thermal misfit strain for all the specimens of sets $A$ and $B$. A sensitivity analysis could be carried out to investigate the sensitivity for these assumptions but is omitted for brevity.

\section{References}

[1] Evans, A. G., Ruhle, M., Dalgleish, B. J., and Charalambides, P. G., 1990 "The Fracture Energy of Bimaterial Interfaces," Mater. Sci. Eng., A, 126, pp. 53-64.

[2] Hutchinson, J. W., and Suo, Z., 1992, "Mixed-Mode Cracking in Layered Materials," Adv. Appl. Mech., 29, pp. 63-191.

[3] Dauskardt, R., Lane, M., Ma, Q., and Krishna, N., 1998, "Adhesion and Debonding of Multi-Layer Thin Film Structures," Eng. Fract. Mech., 61(1), pp. 141-162.

[4] Thouless, M. D., 1990, "Fracture of a Model Interface Under Mixed-Mode Loading," Acta Metall. Mater., 38(6), pp. 1135-1140.

[5] Hutchinson, J. W., 1990, "Mixed Mode Fracture Mechanics of Interfaces," Metal-Ceramic Interfaces, Acta-Scripta Metallurgica Proceedings, Series 4, M. Rühle, et al., eds. Pergamon Press, New York.

[6] Charalambides, P. G., Lund, J., Evans, A. G., and McMeeking, R. M., 1989, "A Test Specimen for Determining the Fracture Resistance of Bimaterial Interfaces," ASME J. Appl. Mech., 56, pp. 77-82.

[7] Odowd, N. P., Shih, C. F., and Stout, M. G., 1992, "Test Geometries for Measuring Interfacial Fracture-Toughness,” Int. J. Solids Struct., 29(5), pp. $571-589$.

[8] Cao, H. C., and Evans, A. G., 1989, "An Experimental-Study of the FractureResistance of Bimaterial Interfaces," Mech. Mater., 7(4), pp. 295-304.

[9] Shih, C. F., 1991, "Cracks on Bimaterial Interfaces-Elasticity and Plasticity Aspects," Mater. Sci. Eng., A, 143(1-2), pp. 77-90.

[10] Wang, J.-S., 1995, "Interfacial Fracture Toughness of a Copper-Alumina System and the Effect of the Loading Phase Angle," Mech. Mater., 20(3), pp. 251-259.

[11] Wang, J. S., and Suo, Z., 1990, "Experimental-Determination of Interfacial Toughness Curves Using Brazil-Nut-Sandwiches," Acta Metall. Mater., 38(7), pp. 1279-1290.

[12] Ikeda, T., Miyazaki, N., and Soda, T., 1998, "Mixed Mode Fracture Criterion of Interface Crack Between Dissimilar Materials," Eng. Fract. Mech., 59(6), pp. $725-735$.

[13] Yuuki, R., Liu, J. Q., Xu, J. Q., Ohira, T., and Ono, T., 1994, "Mixed-Mode Fracture Criteria for an Interface Crack," Eng. Fract. Mech., 47(3), pp. 367377.

[14] Xu, L. M., and Tippur, H. V., 1995, "Fracture Parameters for Interfacial Cracks-An Experimental-Finite Element Study of Crack-Tip Fields and Crack Initiation Toughness," Int. J. Fract., 71(4), pp. 345-363.

[15] Shanbhag, M. R., Eswaran, K., and Maiti, S. K., 1993, "Measurement of Fracture-Toughness of Bimaterial Interfaces and a Stress-Based Approach to Their Fracture," Eng. Fract. Mech., 44(1), pp. 75-89.

[16] Dollhofer, J., Beckert, W., Lauke, B., and Schneider, K., 2000, "Fracture Mechanical Characterisation of Mixed-Mode Toughness of Thermoplast/Glass Interfaces," Comput. Mater. Sci., 19(1-4), pp. 223-228.
[17] Wang, C. H., 1997, "Fracture of Interface Cracks Under Combined Loading," Eng. Fract. Mech., 56(1), pp. 77-86.

[18] Madhusudhana, K. S., and Narasimhan, R., 2002, "Experimental and Numerical Investigations of Mixed Mode Crack Growth Resistance of a Ductile Adhesive Joint," Eng. Fract. Mech., 69(7), pp. 865-883.

[19] Yeung, D. T. S., Yuen, M. M. F., Lam, D. C. C., and Chan, P. C. H., 2000, "Measurement of Interfacial Fracture Toughness for Microelectronic Packages," J. Electron. Manuf., 10(2), pp. 139-145.

[20] Williams, M. L., 1959, "The Stresses Around a Fault or Crack in Dissimilar Media," Bull. Seismol. Soc. Am., 49, pp. 199-204.

[21] Rice, J. R., and Sih, G. C., 1965, "Plane Problems of Cracks in Dissimilar Media," ASME J. Appl. Mech., 32(2), pp. 418-423.

[22] Malyshev, B. M., and Salganik, R. L., 1965, "Strength of Adhesive Joints Using Theory of Cracks," Int. J. Fract. Mech., 1(2), pp. 114-128.

[23] Rice, J. R., 1988, "Elastic Fracture Mechanics Concepts for Interfacial Cracks," ASME J. Appl. Mech., 55, pp. 98-103.

[24] Rice, J. R., Suo, Z., and Wang, J.-S., 1990, "Mechanics and Thermodynamics of Brittle Interfacial Failure in Bimaterial Systems," Acta-Scripta Metallurgica Proceedings Series, M. Ruhle, et al., ed, Pergamon Press, London, pp. 269294.

[25] Suo, Z. G., and Hutchinson, J. W., 1990, "Interface Crack Between 2 Elastic Layers," Int. J. Fract., 43(1), pp. 1-18.

[26] Toya, M., 1992, "On Mode-I and Mode-II Energy-Release Rates of an Interface Crack," Int. J. Fract., 56(4), pp. 345-352.

[27] Agrawal, A., and Karlsson, A. M., 2006, "Obtaining Stress Intensity FactorBased Mode Mixity for a Bimaterial Interface Crack Using the Virtual Crack Closure Technique," Int. J. Fract., 141, pp. 75-98.

[28] Beuth, J. L., 1996, "Separation of Crack Extension Modes in Orthotropic Delamination Models," Int. J. Fract., 77(4), pp. 305-321.

[29] Comninou, M., 1977, "Interface Crack," ASME J. Appl. Mech., 44(4), pp. 631-636.

[30] Matos, P. P. L., McMeeking, R. M., Charalambides, P. G., and Drory, M. D., 1989, "A Method for Calculating Stress Intensities in Bimaterial Fracture," Int. J. Fract., 40(4), pp. 235-254.

[31] Lu, H., and Chiang, F. P., 1993, "Photoelastic Determination of Stress Intensity Factor of an Interfacial Crack in a Bi-Material,” ASME J. Appl. Mech., 60(1) pp. 93-100.

[32] Wang, Y. Y., Chiang, F. P., Barsoum, R. S., and Chou, S. T., 1993, "Study of Deformation Field of Interface Crack in Adhesive Joint," Eng. Fract. Mech., 44(2), pp. 175-184.

[33] Comninou, M., 1990, “An Overview of Interface Cracks," Eng. Fract. Mech., 37(1), pp. 197-208.

[34] Toya, M., 1990, "Fracture-Mechanics of Interfaces," JSME Int. J., Ser. I 33(4), pp. 413-424.

[35] Borovkov, A., Palmov, V., Banichuk, N., Saurin, V., Barthold, F., and Stein, E., 2000, "Macro-Failure Criterion for the Theory of Laminated Composite Structures With Free Edge Delaminations," Comput. Struct., 76(1-3), pp. 195-204

[36] Cook, T. S., and Erdogan, F., 1972, "Stresses in Bonded Materials With a Crack Perpendicular to Interface,” Int. J. Eng. Sci., 10(8), pp. 677-697.

[37] Becker, Jr., T. L., McNaney, J. M., Cannon, R. M., and Ritchie, R. O., 1997 "Limitations on the Use of the Mixed-Mode Delaminating Beam Test Specimen: Effects of the Size of the Region of K-Dominance," Mech. Mater., 25, pp. 291-308.

[38] Broek, D., 1986, Elementary Engineering Fracture Mechanics, Kluwer, Dordrecht.

[39] Agrawal, A., and Karlsson, A. M. 2006, "Mode Mixity Issues in Interfacial Toughness Measurement of Bilayer Specimens," SAMPE 06, Long Beach, CA.

[40] Klingbeil, N. W., and Beuth, J. L., 1997, "Interfacial Fracture Testing of Deposited Metal Layers Under Four-Point Bending," Eng. Fract. Mech., 56(1), pp. 113-126.

[41] Howard, S. J., Phillipps, A. J., and Clyne, T. W., 1993, "The Interpretation of Data From the 4-Point Bend Delamination Test to Measure Interfacial Fracture-Toughness," Composites, 24(2), pp. 103-112.

[42] Charalambides, P. G., Cao, H. C., Lund, J., and Evans, A. G., 1990, "Development of a Test Method for Measuring the Mixed-Mode Fracture-Resistance of Bimaterial Interfaces," Mech. Mater., 8(4), pp. 269-283.

[43] Drory, M. D., Thouless, M. D., and Evans, A. G., 1988, "On the Decohesion of Residually Stressed Thin-Films,” Acta Metall., 36(8), pp. 2019-2028.

[44] ABAQUS, ABAQUS 6.5. 2004, ABAQUS Inc., Pawtucket, RI.

[45] Yang, W., and Shih, C. F., 1994, "Fracture Along an Interlayer," Int. J. Solids Struct., 31(7), pp. 985-1002.

[46] Compston, P., Jar, P. Y. B., Burchill, P. J., and Takahashi, K., 2002, "The Transfer of Matrix Toughness to Composite Mode I Interlaminar Fracture Toughness in Glass-Fibre/Vinyl Ester Composites," Appl. Compos. Mater. 9(5), pp. 291-314. 